\title{
Envelope tracking of a radio frequency amplifier for Long Term Evolution using a three-level class-G modulator
}

\author{
A. Bräckle, L. Rathgeber, F. Siegert, S. Heck, and M. Berroth \\ University of Stuttgart, Institute of Electrical and Optical Communications Engineering, Stuttgart, Germany \\ Correspondence to: A. Bräckle (alexander.braeckle@int.uni-stuttgart.de)
}

\begin{abstract}
In this contribution, efficiency enhancement of a radio frequency $(\mathrm{RF})$ power amplifier (PA) for Long Term Evolution (LTE) base stations using envelope tracking is investigated. The dynamically adapted drain bias voltage is provided by a three-level class-G modulator. The potential of this bias modulator is demonstrated with a class AB PA built in gallium nitride $(\mathrm{GaN})$ technology with an operation frequency of $2.75 \mathrm{GHz}$, a maximum output power of $41 \mathrm{dBm}$ and a peak power-added efficiency (PAE) of $60 \%$. When driven by an LTE signal with a bandwidth of $20 \mathrm{MHz}$, overall system efficiency can be increased from $17 \%$ with a constant supply voltage up to $29 \%$ with envelope tracking. This is accompanied by a reduction of linearity. Adjacent channel leakage ratio (ACLR) decreases by $10 \mathrm{~dB}$ in the first and by $18 \mathrm{~dB}$ in the second adjacent channel, respectively. Error vector magnitude (EVM) increases from $9 \%$ up to $29 \%$.
\end{abstract}

\section{Introduction}

In an RF transmitter, by far most of the power is consumed by the PA. Typically, more than $50 \%$ of the base station input power is dissipated in this component (Blume et al., 2010). Therefore, efficiency enhancement of RF PAs is in the focus of many research activities. In this contribution, PAE is considered to quantify efficiency. It is defined as

$\mathrm{PAE}=\frac{P_{\mathrm{out}}-P_{\text {in }}}{P_{\mathrm{DC}}}$,

where $P_{\text {out }}$ is the RF output power of the PA, $P_{\text {in }}$ denotes the RF input power, and $P_{\mathrm{DC}}$ is the DC power consumed by the PA. PAs can be operated very efficiently when driven in the compression region, but PAE rapidly drops for low output powers (Cripps, 1999).

This poses severe challenges especially for modern mobile communications systems such as LTE. Due to the con- stantly increasing bandwidth requirements, these systems utilize higher-order modulation schemes resulting in a high peak-to-average power ratio (PAPR) of $10 \mathrm{~dB}$ and more. Apart from efficiency, linearity is a second characteristic to be considered in order to avoid distortion of the transmitted signal. The mobile communications standards pose obligatory linearity requirements on all components in transmitters (ETSI, 2011). Therefore, nowadays mainly PAs of classes A, $\mathrm{AB}$, and $\mathrm{B}$ are in use due to their high linearity. However, average efficiency is in the order of only about $20 \%$.

Several concepts for efficiency enhancement without reducing amplifier linearity are currently investigated, such as the Doherty amplifier (Doherty, 1936), the Chireix amplifier (Chireix, 1935), Class-S amplifiers (Raab, 1973), and Envelope Tracking (ET). The latter one is studied in more detail in this paper.

\section{Envelope tracking}

The concept of ET was first proposed by Saleh and Cox (1983). In order to understand the principle of this idea, the drain-source voltage $u_{\text {DS }}$ of the transistor in a PA with an amplitude-modulated input signal is considered. As it can be seen in Fig. 1, $u_{\mathrm{DS}}$ oscillates around the drain bias voltage $U_{\mathrm{DC}}$. For high output powers the available voltage swing between $0 \mathrm{~V}$ and $U_{\mathrm{DC}}$ is fully used. However, for lower output powers, the amplitude of $u_{\mathrm{DS}}$ decreases, resulting in higher losses. Those can be represented by the area below the envelope of the drain-source voltage.

The idea of ET is to dynamically adapt the drain bias voltage to the instantaneous output power of the PA. The corresponding drain-source voltage is depicted in Fig. 2. The area below the envelope of $u_{\mathrm{DS}}$ is minimised, resulting in an increased efficiency of the circuit. 


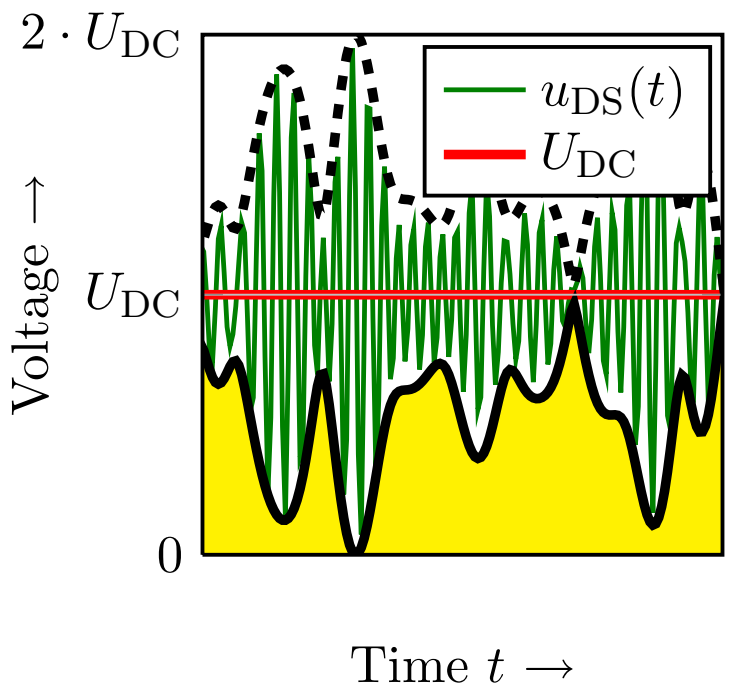

Fig. 1. Drain-source voltage of a PA with fixed drain bias.

\section{Class-G amplifier architecture}

There are several architectures for voltage modulators. Due to the high efficiency requirements, they are all based on switch-mode topologies.

Buck modulators are pure switch-mode converters. In order to generate a continuous-valued drain bias voltage, the desired waveform is generated by pulse-width modulation (PWM) and subsequent low-pass filtering. As PWM is based on oversampling, the switching frequency needs to be significantly higher than the bandwidth of the output signal. For broadband signals such as LTE, this results in switching frequencies above $100 \mathrm{MHz}$, leading to high switching losses for large power transistors. Therefore, buck converters with output powers above $2 \mathrm{~W}$ are either used in narrow-band applications (Raab, 1998; Høyerby and Andersen, 2007, 2009) or implemented as integrated circuits in RF power technologies such as LDMOS (Pinon et al., 2008) or GaAs (Busking et al., 2012).

In switch-mode assisted linear modulators, a linear stage and a switching stage are operated in parallel. When properly designed, the main portion of the modulator output current is provided by the switching stage. The linear stage works as an active filter and compensates for the ripple generated by the switching stage. Therefore, the switching frequency can be chosen much lower and switching losses are reduced. Switch-mode assisted linear modulators are widely used (Kimball et al., 2006; Jeong et al., 2009; Hoversten and Popovic, 2010), but suffer from two disadvantages. On the one hand, for very high output powers, the absolute power dissipated in the linear stage becomes very high even if it is small compared to the output power. This poses severe challenges on the design of a broadband linear stage. On the other hand, modulator efficiency drops for low output powers due
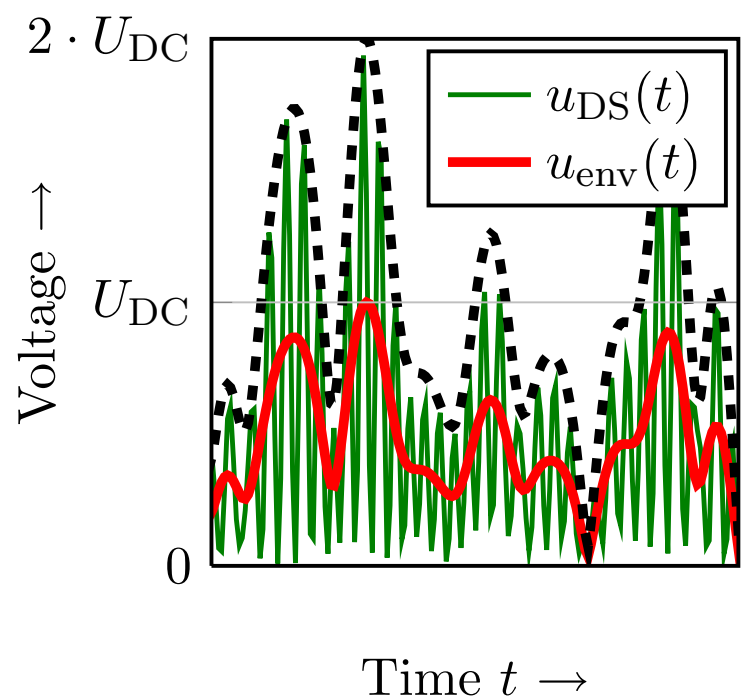

Fig. 2. Drain-source voltage of a PA in an ET system.

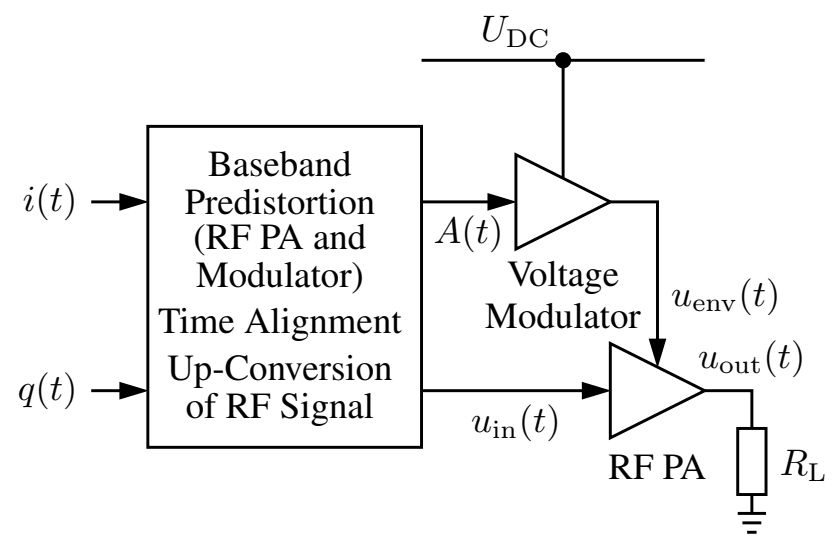

Fig. 3. Block diagram of an ET system.

to the inherent switching operation, which results in constant switching losses.

One way to overcome these drawbacks is the class-G concept, which was first proposed by Sampei et al. (1978) for audio amplifiers and then implemented for RF applications by Raab (1986). In contrast to the modulators introduced above, the drain bias can only be set to discrete-valued voltage levels. The simplest configuration of a two-level class-G modulator is depicted in Fig. 4. If the envelope $A(t)$ is above a fixed reference voltage $U_{\text {ref, } 1}$, the transistor $T_{1}$ is turned on and the modulator output voltage $u_{\text {env }}$ equals the high supply voltage $U_{\mathrm{DC}, \mathrm{M}_{+}}$. For low envelopes, the transistor is in off-state and the diode $D_{1}$ connects the low supply voltage $U_{\mathrm{DC}, \mathrm{M}-}$ to the output.

Although the RF PA does not work in its highest-efficiency operating point most of the time when using a class-G modulator, overall system efficiency can be significantly increased in the backoff region. Furthermore, the voltage modulator 


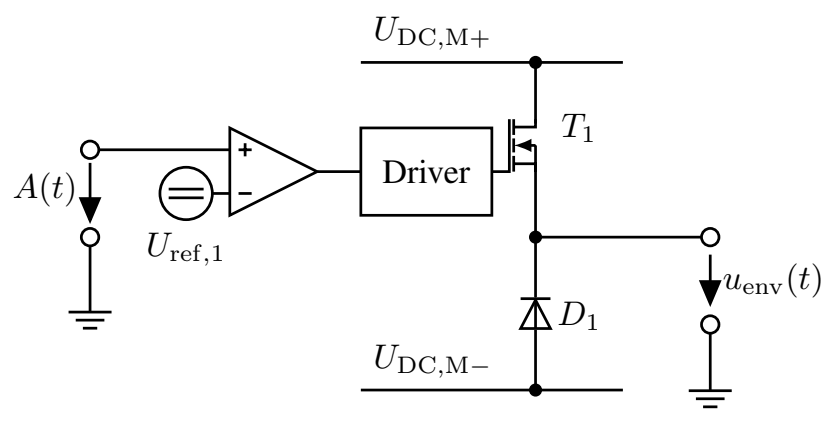

Fig. 4. Simplified circuit diagram of a two-level class-G modulator.

can be designed very efficiently due to its simplicity. Especially at low modulator output voltages, very high efficiencies can be achieved because no inherent switching occurs.

The efficiency can be improved further by inserting more than two discrete voltage levels. In this work, a three-level class-G modulator is presented.

\section{Radio frequency power amplifier}

The potential of multi-level class-G modulators is demonstrated with an RF PA based on a gallium nitride $(\mathrm{GaN})$ high electron mobility transistor (HEMT). The device is the commercially available CGH40010 manufactured by Cree and has a nominal supply voltage of $28 \mathrm{~V}$. With this transistor, a class $\mathrm{AB}$ PA operating at a center frequency of $2.75 \mathrm{GHz}$ is designed.

Figure 5 shows the measured PAE of the PA with a fixed drain bias voltage of $28 \mathrm{~V}$ when driven by a continuous-wave (CW) input signal. The amplifier achieves a peak PAE of $60 \%$ at an output power of $41 \mathrm{dBm}$. However, as expected, efficiency drops in the backoff region. As derived by Cripps (1999), PAE is halved for a reduction of output power by approximately $6 \mathrm{~dB}$.

Furthermore, Fig. 5 also depicts the probability density function (PDF) of an LTE signal. It can be seen that the PA is operated at an output power of about $29 \mathrm{dBm}$ most of the time under this excitation. At this output power, efficiency with a CW signal drops below $20 \%$. With a dynamic LTE input signal, average efficiency is $22.4 \%$, which is significantly less than the maximum PAE.

The potential of the class-G architecture becomes clear when performing CW measurements of the RF PA with reduced supply voltages. Figure 5 shows the PAE versus output power not only for the maximum supply voltage of $28 \mathrm{~V}$, but also for $U_{\mathrm{DC}}=10 \mathrm{~V}$ and $U_{\mathrm{DC}}=20 \mathrm{~V}$. For lower supply voltages, the point of peak PAE is shifted towards lower output powers. For instance, PAE in CW operation can be increased up to about $44 \%$ at an output power of $29 \mathrm{dBm}$ when reducing the drain bias to $10 \mathrm{~V}$.

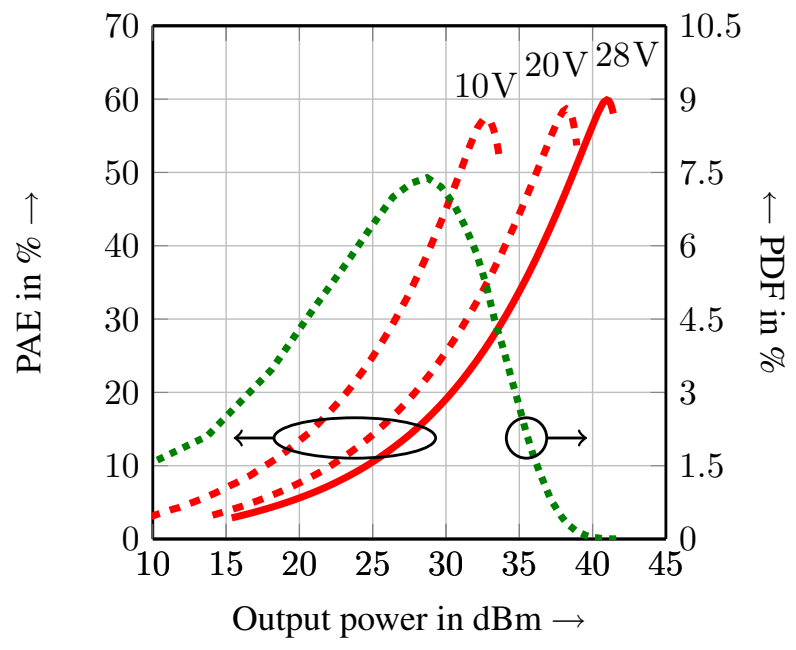

Fig. 5. Measured PAE of the PA under CW excitation for a fixed supply voltage of $28 \mathrm{~V}$ (solid), reduced supply voltages (dashed) and PDF of an LTE signal (dotted).

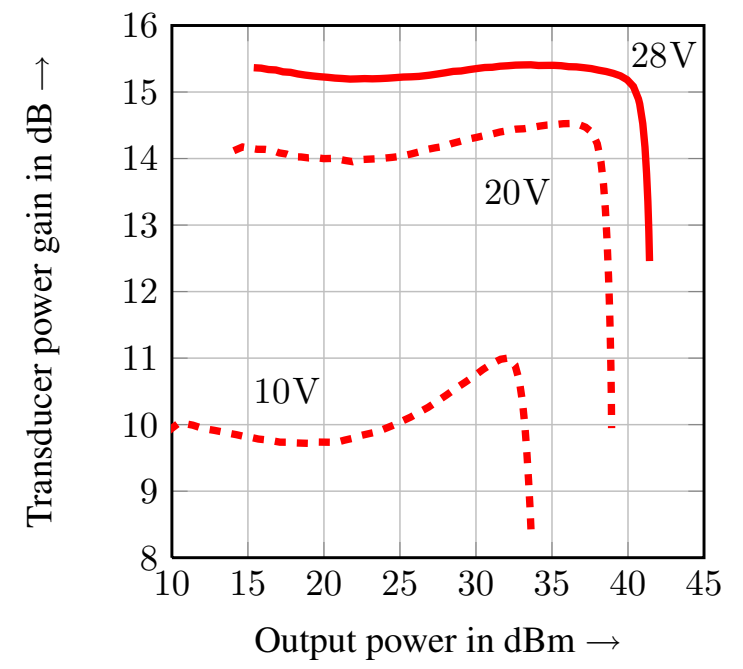

Fig. 6. Measured transducer power gain of the PA under CW excitation for several supply voltages.

However, the supply voltage of the PA cannot be arbitrarily reduced. As shown in Fig. 6, the transducer power gain of the $\mathrm{PA}$, which is defined by

$G_{\mathrm{T}}=\frac{P_{\text {out }}}{P_{\mathrm{avs}}}$

where $P_{\text {avs }}$ denotes the available source power, drops for lower supply voltages. In this work, $G_{\mathrm{T}}$ shall not be lower than $10 \mathrm{~dB}$. This leads to an allowable drain bias range from $10 \mathrm{~V}$ to $28 \mathrm{~V}$. 


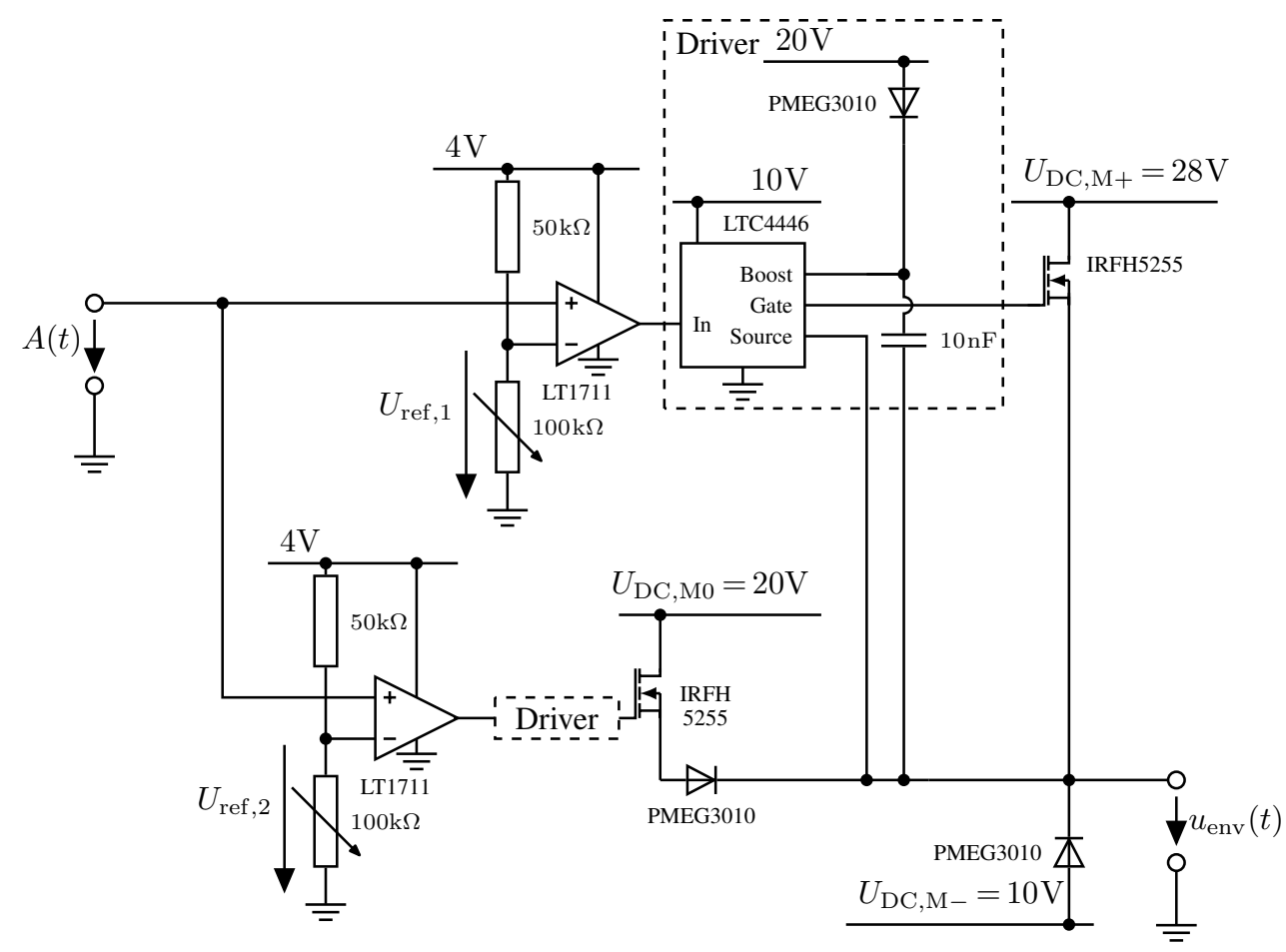

Fig. 7. Schematic of the designed three-level class-G modulator. For the sake of simplicity, shunt capacitors in the supply lines are omitted.

\section{Class-G modulator design}

Based on the analysis of the RF PA in the previous section, design requirements for the three-level class-G voltage modulator can be deduced. The highest drain bias voltage has to be equal the nominal value, that is $U_{\mathrm{DC}, \mathrm{M}+}=28 \mathrm{~V}$. For the low supply voltage, $U_{\mathrm{DC}, \mathrm{M}_{-}}=10 \mathrm{~V}$ are chosen, which makes sure that gain does not drop below $10 \mathrm{~dB}$. The third voltage level $U_{\mathrm{DC}, \mathrm{M} 0}$ has to be between those two. In this work, it is arbitrarily chosen to be $U_{\mathrm{DC}, \mathrm{M} 0}=20 \mathrm{~V}$. Furthermore, the modulator needs to be able to deliver a maximum output current of $1 \mathrm{~A}$, which is the supply current required by the PA. A high switching speed is also desirable in order to follow the envelope of an LTE signal with a bandwidth of $20 \mathrm{MHz}$.

The schematic of the designed class- $G$ modulator is depicted in Fig. 7. It consists of two identical paths to control the power transistors switching the discrete supply voltages. The envelope $A(t)$ of the RF signal, which serves as input signal of the circuit, is compared to constant reference volt-

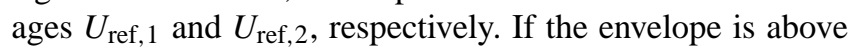
the reference level, a subsequent gate driver circuit turns on the associated power transistor and thus connects $U_{\mathrm{DC}, \mathrm{M}+}$ or $U_{\mathrm{DC}, \mathrm{M} 0}$ to the modulator output node. If $A(t)$ is smaller than both reference voltages, none of the transistors is turned on, the diode attached to the output node conducts and $u_{\text {env }}$ equals $U_{\mathrm{DC}, \mathrm{M}-}$.
For the power transistors, $\mathrm{n}$-channel metal-oxide semiconductor field-effect transistors (MOSFETs) are used due to their higher switching speed compared to p-channel MOSFETs. As they are controlled by the gate-source voltage and due to the fact that the gate is connected to the output and thus has no fixed potential, a gate driver integrated circuit (IC) is used in a bootstrap configuration.

\section{Measurement results}

In this section, the measurement results achieved when operating the RF PA with a dynamic supply voltage generated by the three-level voltage modulator are presented. The generation of the modulator supply voltages is not included in the efficiency calculations. A photograph of the circuit setup can be seen in Fig. 8 .

\subsection{Static measurements}

First, measurements under CW excitation are performed. In this case, one of the three possible supply voltages is constantly turned on by the modulator and the power of the RF PA input signal is increased.

The measured PAE of the entire system is depicted in Fig. 9. The efficiency for a constant supply voltage of $28 \mathrm{~V}$ is drawn as well for comparison. It can be clearly seen that efficiency in the backoff region is significantly increased by the class-G modulator. For output powers up to $33 \mathrm{dBm}$, the 


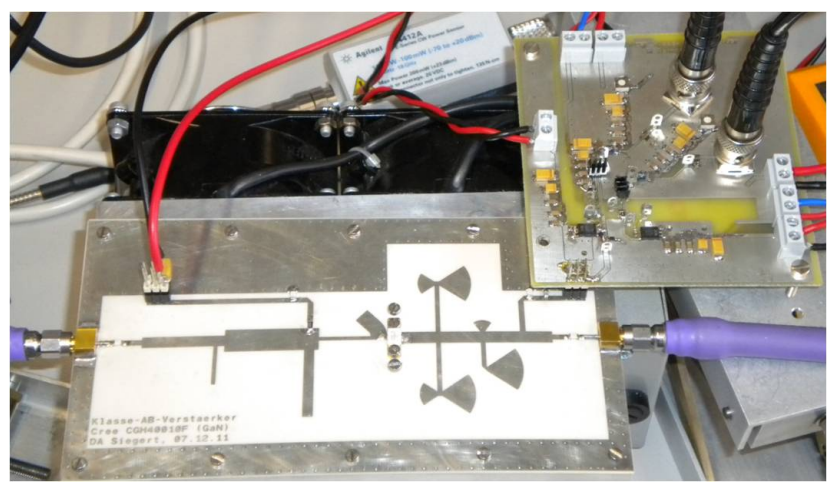

Fig. 8. Class-G modulator (top right) connected to the RF PA (bottom left).

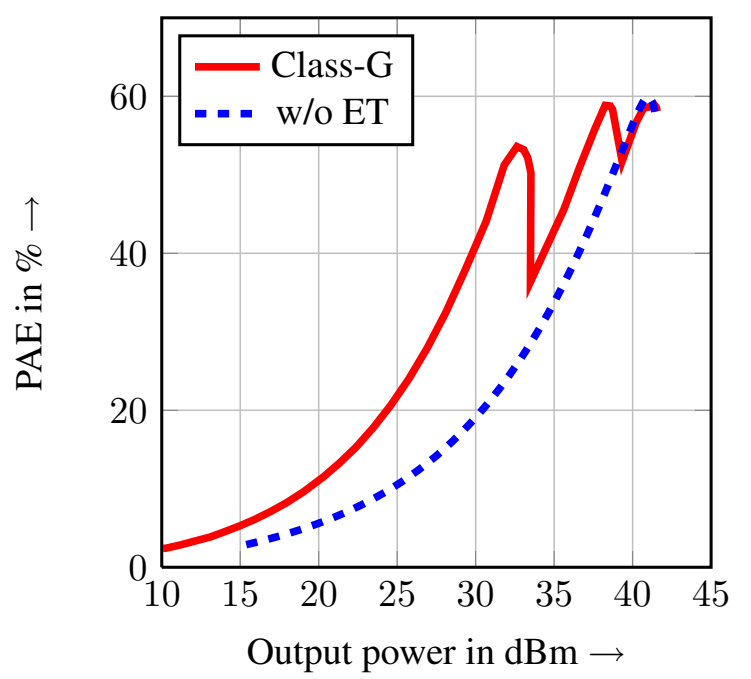

Fig. 9. Measured PAE of the entire class-G system under CW excitation.

PA is supplied by the low bias voltage $U_{\mathrm{DC}, \mathrm{M}-}=10 \mathrm{~V}$. For output powers between $33 \mathrm{dBm}$ and $38 \mathrm{dBm}$, the drain bias is set to $U_{\mathrm{DC}, \mathrm{M} 0}=20 \mathrm{~V}$. If the output power is even higher, $u_{\mathrm{env}}$ equals $U_{\mathrm{DC}, \mathrm{M}+}=28 \mathrm{~V}$. By this, PAE at an output power of $29 \mathrm{dBm}$ can be increased from $17 \%$ up to almost $38 \%$.

\subsection{Dynamic measurements}

The class-G system is also characterised with excitation by an LTE signal at the input. It has a bandwidth of $20 \mathrm{MHz}$ and a PAPR of $11.6 \mathrm{~dB}$. The class- $G$ modulator is driven by the according envelope $A(t)$. The reference voltages are computed by

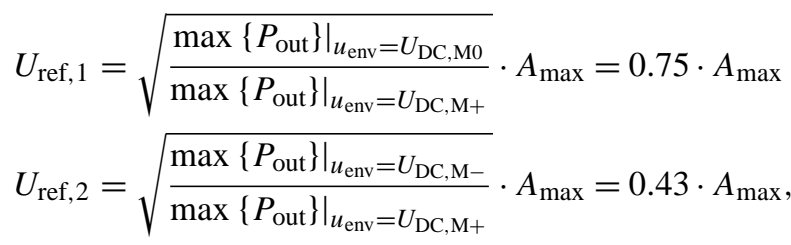

Table 1. Measured efficiency and linearity of the class-G system under LTE excitation.

\begin{tabular}{lcccc}
\hline & PAE & ACLR1 & ACLR2 & EVM \\
\hline Requirement & & $-44.2 \mathrm{~dB}$ & $-44.2 \mathrm{~dB}$ & $9.0 \%$ \\
w/o ET & $16.7 \%$ & $-27.2 \mathrm{~dB}$ & $-43.9 \mathrm{~dB}$ & $10.0 \%$ \\
3-level class-G & $29.2 \%$ & $-17.9 \mathrm{~dB}$ & $-26.1 \mathrm{~dB}$ & $29.3 \%$ \\
2-level class-G & $32.2 \%$ & $-18.9 \mathrm{~dB}$ & $-24.8 \mathrm{~dB}$ & $31.6 \%$ \\
\hline
\end{tabular}

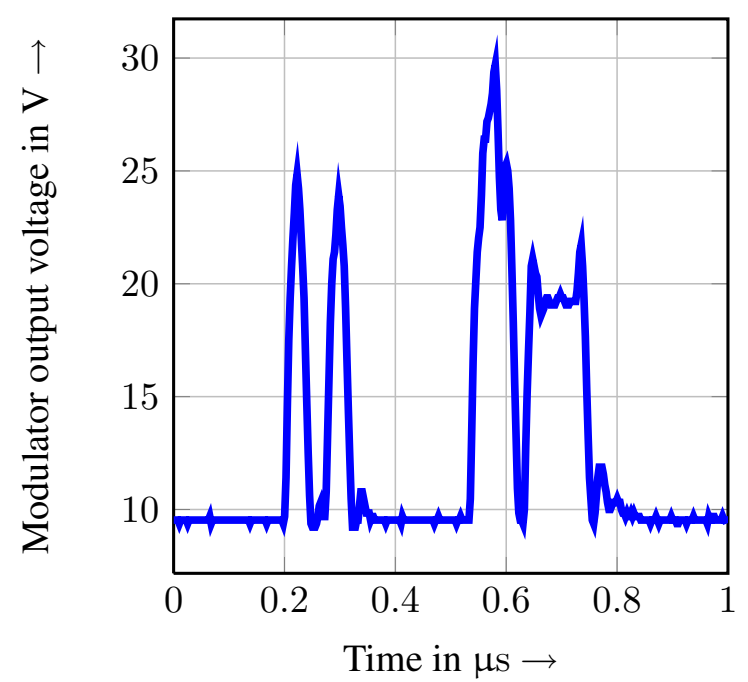

Fig. 10. Time-domain measurement of the modulator output voltage with LTE excitation.

where $A_{\max }$ denotes the maximum of the modulator input signal.

Figure 10 shows a time-domain measurement of the modulator output voltage. The results are summarized in Table 1. For comparison, measurement results of a two-level class-G modulator presented in (Bräckle et al., 2012) are included as well. Efficiency can be enhanced from $16.7 \%$ up to $29.2 \%$ by using the three-level modulator, which is a significant increase. However, in contrary to theory, PAE of the two-level configuration is even slightly higher. This is caused by the additional circuitry required to drive two switches. Furthermore, in the present three-level configuration, both switches are turned on at high output powers although only the MOSFET for $U_{\mathrm{DC}, \mathrm{M}+}$ is needed. This can be overcome by a digital control circuit. And finally, the medium supply voltage $U_{\mathrm{DC}, \mathrm{M} 0}$ is arbitrarily chosen to be $20 \mathrm{~V}$. A further increase in efficiency is expected after optimization of $U_{\mathrm{DC}, \mathrm{M} 0}$ with respect to the statistical properties of an LTE signal.

In contrast, linearity decreases. ACLR in the first channel reduces by about $10 \mathrm{~dB}$, in the second channel it is even $17 \mathrm{~dB}$ worse than for a constant supply voltage. EVM is $29.3 \%$, which is three times the value with a fixed drain bias. For the two-level configuration, linearity is even worse. This is mainly caused by the shift of gain for different supply 
voltages shown in Fig. 6. Every time $u_{\text {env }}$ is changed, the gain changes as well, resulting in an additional amplitude modulation and distorting the signal.

\section{Conclusions}

This work has presented the potential as well as the challenges in designing multi-level class-G systems. By using a three-level modulator, overall system efficiency can be enhanced from $16.7 \%$ up to $29.2 \%$. However, linearity is significantly degraded, which is mainly caused by the supply voltage dependency of the RF PA gain. Therefore, in order to benefit from the efficiency enhancement of class-G modulators, RF PAs need to be optimized for constant gain at several supply voltages.

Further optimizations are to be carried out on an intelligent driver system to avoid that two transistors are turned on at the same time. An optimized choice of the voltage levels will be investigated as well.

\section{References}

Blume, O., Zeller, D., and Barth, U.: Approaches to Energy Efficient Wireless Access Networks, in: 4th International Symposium on Communications, Control and Signal Processing, Limassol, Cyprus, 3-5 March 2010, 1-5, 2010.

Bräckle, A., Rathgeber, L., Siegert, F., Heck, S., and Berroth, M.: Power Supply Modulation for RF Applications, in: 15th International Power Electronics and Motion Control Conference, Novi Sad, Serbia, September 2012.

Busking, E., de Hek, P., and van Vliet, F.: 1 GHz GaAs Buck Converter for High Power Amplifier Modulation Applications, in: Proceedings of the 7th European Microwave Integrated Circuits Conference, 353-356, Amsterdam, October 2012.

Chireix, H.: High Power Outphasing Modulation, P. IRE, 23, 13701392, 1935.

Cripps, S.: RF Power Amplifiers for Wireless Communications, 1st edition, Arctech House, Boston, London, 1999.

Doherty, W.H.: A New High Efficiency Power Amplifier for Modulated Waves, P. IRE, 24, 1163-1182, 1936.
Evolved Universal Terrestrial Radio Access (E-UTRA); LTE: Base Station (BS) conformance testing (3GPP TS 36.141 version 9.6.0 Release 9), European Telecommunications Standards Institute, 2011.

Hoversten, J. and Popovic, Z.: System Considerations for Efficient and Linear Supply Modulated RF Transmitter, in: IEEE 12th Workshop on Control and Modeling for Power Electronics, Boulder, June 2010.

Høyerby, M. and Andersen, M.: Self-Oscillating Soft Switching Envelope Tracking Power Supply for Tetra2 Base Station, in: 29th International Telecommunications Energy Conference, 5360, September 2007.

Høyerby, M. and Andersen, M.: TEDS Base-Station Power Amplifier Using Low-Noise Envelope Tracking Power Supply, IEEE T. Microw. Theory, 57, 1687-1693, 2009.

Jeong, J., Kimball, D., Kwak M., Draxler P., Hsia C., Steinbeiser, C., Landon, T., Krutko, O., Larson, L., and Asbeck, P.: HighEfficiency WCDMA Envelope Tracking Base-Station Amplifier Implemented With GaAs HVHBTs, IEEE J. Solid-St. Circ., 44, 2629-2639, 2009.

Kimball, D., Jeong, J., Hisa, C., Draxler, P., Lanfranco, S., Nagy, W., Linthicium, K., Larson, L., and Asbeck, P.: High-Efficiency Envelope-Tracking W-CDMA Base-Station Amplifier Using GaN HFETs, IEEE T. Microw. Theory, 54, 3848-3856, 2006.

Pinon, V, Hasbani, F., Giry, A., Pache, D., and Garnier, C.: A Single-Chip WCDMA Envelope Reconstruction LDMOS PA with $130 \mathrm{MHz}$ Switched-Mode Power Supply, in: IEEE Int. Solid-St. Circ. Conf., 564-636, 2008.

Raab, F. H.: Radio Frequency Pulsewidth Modulation, IEEE T. Commun., 21, 958-966, 1973.

Raab, F.: Average Effciency of Class-G Power Amplifiers, IEEE T. Consum. Electr., 32, 145-150, 1986.

Raab, F., Sigmon, B., Myers, R., and Jackson, R.: L-Band Transmitter Using Kahn EER Technique, IEEE T. Microw. Theory, 46, 2220-2225, 1998.

Saleh, A. A. M. and Cox, D. C.: Improving the Power-Added Efficiency of FET Amplifiers Operating with Varying-Envelope Signals, IEEE T. Microw. Theory, 31, 51-56, 1983.

Sampei, T., Ohashi, S., Ohta, Y., and Inoue, S.: Highest Efficiency and Super Quality Audio Amplifier Using MOS Power FETs in class G Operation, in: IEEE T. Consum. Electr., 24, 300-307, 1978. 